\title{
A Mobility Model for Cost Analysis in Integrated Cellular/WLANs
}

\author{
Nirmala Shenoy, Bruce Hartpence, \\ Information Technology Department, Rochester Institute of Technology, Rochester NY 14623, USA \\ Rafael Mantilla Montalvo, Ph.D., \\ Mobile Wireless Group, Cisco Systems, USA.
}

\begin{abstract}
In this article, we propose a model that can be used to study the costs and benefits of integrating cellular and Wireless LANs, from a vendor and a user perspective. We explain the model and the approach to calculate the costs for a simple handoff topology that supports a voice call across a cellular and a WLAN. The mobility model, which can be used to estimate the probability of a cellular user entering a WLAN while moving within the "Location Area" of the cellular network, is the main contribution of this work. The mobility model takes into account a normal user movement and a biased user movement based on a 2D random walk model. We show how this model could be used to study the costs and benefits to the vendor and the user, especially if a user were to be supported with call continuity across the two networks and the vendor had to bear the extra costs of handing over an active call. We have restricted our analysis to a scenario where the user with an active voice call moves from the cellular network to the WLAN. Graphs and discussions are provided to demonstrate the use of the model to study the integration benefits, from a vendor and a user perspective.
\end{abstract}

\section{INTRODUCTION}

The idea of an integrated cellular and wireless LAN for service support is very appealing to both the vendor and the mobile user community. The complementary features of the two technologies are the prime reason for this. The authors are currently working on a framework to support global roaming across heterogeneous wireless networks. Part of the framework $^{1}$ study involves the cost/benefits analysis of such an integrated setup. In this paper, we introduce a model, which can be used to estimate the cost/benefits in integrating these two technologies from both the user and the vendor perspective. Though a number of case studies would arise under such an integrated scenario, we restrict the presentation to the application of the model and associated cost analysis to support a voice call across the two networks. (The infrastructure costs are not taken into consideration.) To our knowledge, there is no such model available to date for such studies and hence we do not provide any comparisons. The model is flexible and can be extended to hexagonal cells, different WLAN coverage areas and overlapped cells.

We conduct studies using two mobility models - an unbiased mobility model and a biased mobility model. The biased mobility model takes into consideration the preferred user movement, if he is aware of a cheaper WLAN option available in the vicinity.

\footnotetext{
${ }^{1}$ This work is sponsored and funded under the Cisco URP grant and the Lab for Applied Computing, RIT
}

The next section gives some assumptions made to restrict the scope of the work presented. This is followed by the section which describes a simple handoff topology and the signaling information flow for a voice call handoff. The subsequent section explains the mobility model used. Analysis of the unbiased and the biased models are provided. The section that follows provides some statistics on expected WLAN entries (by roaming cellular users) and introduces some user-mix distributions to be used for the vendor cost analysis. The section that follows provides the cost calculations, graphs and discussion from a vendor and a user perspective. A delay analysis has been added to highlight the effects of such integration on the user perceived delays. The last section provides conclusions and future work.

\section{ASSUMPTIONS}

Due to space considerations we have limited the scope of study and analysis presented. Following were the assumptions made in this regard.

- The cell in the cellular network and the WLAN Access Point (AP) coverage are assumed to be square in shape The WLAN occupies one square cell in the middle of a "Location Area" in the cellular network. No overlap issues are considered. The mobility model has been developed based on these assumptions. Ideas to extend the study to hexagonal cells and overlapped coverage areas can be found in $[1,2]$.

- The Location Area in the cellular network has been restricted to $5 * 5$ square cells and hence is square shaped. This Location Area (LA) is under one Visitor Location Area (VLR) coverage area of the cellular network.

- The Mobile Node (MN) is considered to be in the Home Network. A simple roaming and handoff scheme from cellular to WLAN, with call re-direction occurring at the $\mathrm{MSC} /$ gateway is considered.

- The handoff and associated cost study has been restricted to voice calls. A 'forward' handoff mechanism from the WLAN is considered which could result in loss of some voice packets.

- It is assumed that in the WLAN voice calls will be supported via Voice over IP.

- A unit called URAC (User Registration and Authentication Center) is assumed to accommodate for initiation of any registration requests and authentication by the Home Location Area (HLR) of the cellular network. 


\section{THE HANDOFF TOPOLOGY}

The handoff topology shows the main network elements that will be involved in handing off a voice call from the cellular network to the WLAN. The topology is selfexplanatory. The information flow for this topology implementing a forward handoff is given below in Figure 2. (Authentication and association between $\mathrm{MN}$ and AP are not shown.) The messages are numbered and have selfexplanatory names. Where more than one message performs the same function, the numbers of these messages are appended at the end of the message name. The shaded triangular blocks are points of database access/processing. Message 16 indicated as 'Location Update' informs the HLR that the MN is currently in the WLAN coverage area. The cost associated with each message is given as $c_{x}$ to facilitate the calculation given under section 5

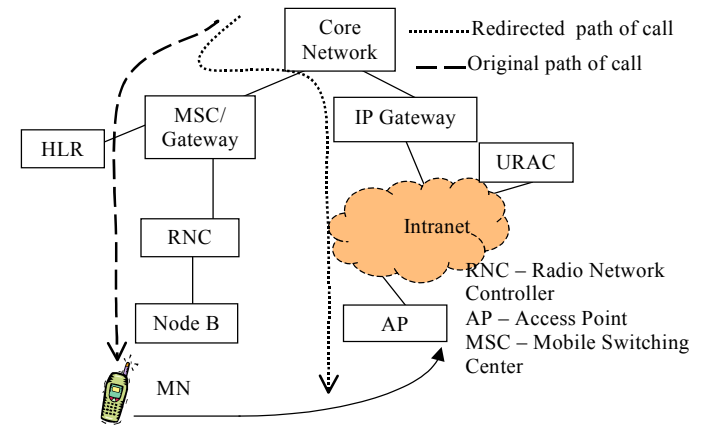

Figure 1. Roaming and Handoff Topology

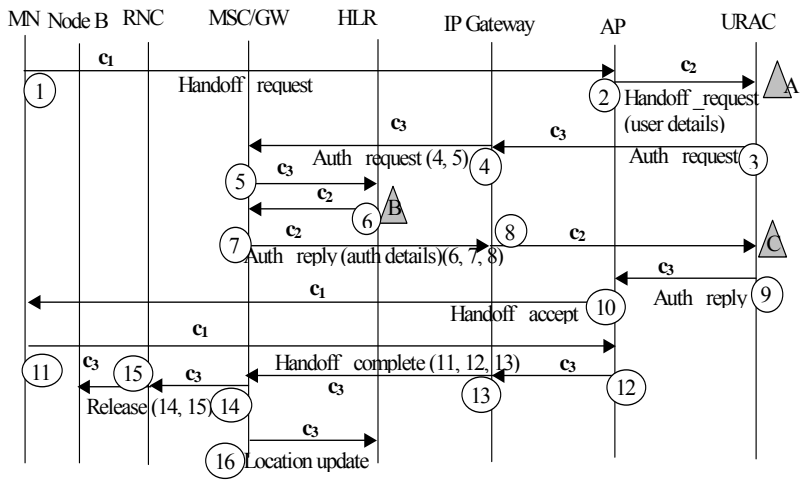

gure 2. Information Flow for Handoff and WLAN Update

IV. THE MOBILITY MODEL

An unbiased and a biased mobility model are introduced and explained in this section. A regular Markov chain model has been used in both cases. This model will provide an estimate on the average number of times that a user will transit into the WLAN cell, while performing a random walk in the LA of the cellular network. This value is necessary to estimate the extra cost incurred by the vendors for signaling and processing handoff between cellular and WLAN.

\section{A. Unbiased Mobility model}

Figure 3 shows the LA cells numbered to help develop the unbiased mobility model. A user in cells with identical numbers will have a similar mobility pattern. Hence, there are six categories of cells, based on the mobility pattern of the user. Cell number 6 is the WLAN cell. Starting from any cell 1,2, 3 etc we are interested in the average number of transitions a user makes into cell 6 i.e. the WLAN cell. All cells in adjacent LA are numbered 7. The state transition diagram for this model is given in figure 4. As this is an unbiased model, the probability of transition from any cell to the adjacent cell is equal to $1 / 4$. Transition into state/cell 6 is indicated by dotted lines. The probability transition matrix for state transition diagram of figure 4 is given in equation 1 .

\begin{tabular}{l|l|l|l|l|l|l}
7 & 7 & 7 & 7 & 7 & 7 & 7 \\
\hline 7 & 1 & 2 & 3 & 2 & 1 & 7 \\
\hline 7 & 2 & 4 & 5 & 4 & 2 & 7 \\
\hline 7 & 3 & 5 & 6 & 5 & 3 & 7 \\
\hline 7 & 2 & 4 & 5 & 4 & 2 & 7 \\
\hline 7 & 1 & 2 & 3 & 2 & 1 & 7 \\
\hline 7 & 7 & 7 & 7 & 7 & 7 & 7
\end{tabular}

Figure 3. Square Cell numbering in the model

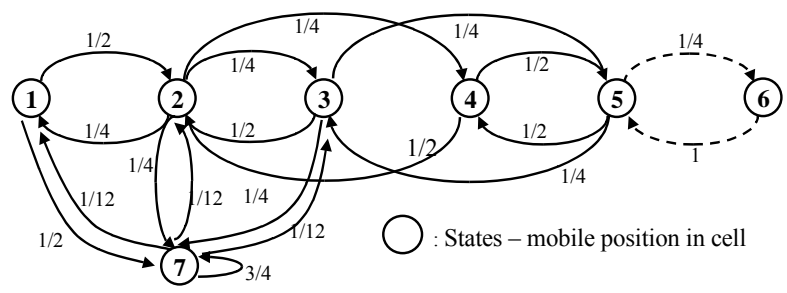

Fig 4, State Transition Diagram WLAN cell entry

$$
\left.\mathbf{P}=\left[\mathrm{p}_{\mathrm{ij}}\right]=\mathrm{l} \begin{array}{lllllll}
1 & 2 & 3 & 4 & 5 & 6 & 7 \\
0 & 1 / 2 & 0 & 0 & 0 & 0 & 1 / 2 \\
1 / 4 & 0 & 1 / 4 & 1 / 4 & 0 & 0 & 1 / 4 \\
0 & 1 / 2 & 0 & 0 & 1 / 4 & 0 & 1 / 4 \\
0 & 1 / 2 & 0 & 0 & 1 / 2 & 0 & 0 \\
0 & 0 & 1 / 4 & 1 / 2 & 0 & 1 / 4 & 0 \\
0 & 0 & 0 & 0 & 1 & 0 & 0 \\
1 / 12 & 1 / 12 & 1 / 12 & 0 & 0 & 0 & 3 / 4
\end{array}\right)
$$

Given the probability transition matrix the average number of times a user roams into the WLAN cell can be calculated. This is given in the next section. When movement into the WLAN cell occurs, location update/ handoff will be initiated.

\section{B. Biased Mobility Model}

Users aware of WLAN coverage in the vicinity would tend to move into this cell, due to the lower costs for voice call and higher bit rate for data connections. Hence, the second model introduces some bias in the user movement. The transition probabilities from cells 4 and 5 are biased towards the WLAN cell 6. (To avoid cluttering the picture all other transitions and cells numbered 7 are not shown in figure 5.) We have selected some transition probabilities that show the biased movement of the user towards the WLAN cell. Other values can be used. The state transition diagram for the biased model will be similar to that in Figure 4 but with different transition rates, hence it is not repeated. The transition probability matrix for the biased model is given in equation 2 . 


\section{Average Number of Crossings into WLAN}

From the transition probability matrix $\mathbf{P}$ (given by equations 1 \&2), the fundamental matrix $\mathrm{Z}$ for the regular Markov chain is given by equation 3 .

$\mathbf{Z}=\left[z_{i \mathrm{ij}}\right]=(\mathbf{I}-\mathbf{P}+\mathbf{A})^{-1}$

In this equation $\mathbf{A}$ is the limiting matrix determined by $\mathbf{P}$, where each row of $\mathbf{A}$ comprises of the same probability vector $\pi=\left\{\pi_{1}, \pi_{2}, \ldots, \pi_{\mathrm{n}}\right\}$, i.e. $\mathbf{A}=\xi \pi$ ( $\xi$ is column vector with all entries equal to 1) and $\mathbf{I}$ is the identity matrix. [5].

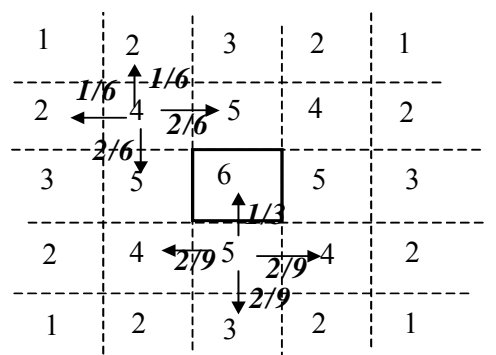

Figure 5. Biased model - Transition Probabilities

\begin{tabular}{|c|c|c|c|c|c|c|c|c|}
\hline & & 1 & 2 & 3 & 4 & 5 & 6 & \\
\hline & 1 & 0 & $1 / 2$ & 0 & 0 & 0 & 0 & $1 / 2$ \\
\hline & 2 & $1 / 4$ & 0 & $1 / 4$ & $1 / 4$ & 0 & 0 & $1 / 4$ \\
\hline $\mathbf{P}=\left[\mathrm{p}_{\mathrm{ij}}\right]$ & 3 & 0 & $1 / 2$ & 0 & 0 & $1 / 4$ & 0 & $1 / 4$ \\
\hline & 4 & 0 & $1 / 3$ & 0 & 0 & $2 / 3$ & 0 & 0 \\
\hline & 5 & 0 & 0 & $2 / 9$ & $4 / 9$ & 0 & $1 / 3$ & 0 \\
\hline & 6 & 0 & 0 & 0 & 0 & 1 & 0 & 0 \\
\hline & 7 & $1 / 12$ & $1 / 12$ & $1 / 12$ & 0 & 0 & 0 & $3 / 4$ \\
\hline
\end{tabular}

Let $\mathrm{y}_{\mathrm{j}}^{(\mathrm{k})}$ be the number of times that a process is in the state $S_{j}$ in the first $k$ steps, then if $M_{i}\left[y_{j}^{(k)}\right]$ is the mean number of times the process is in state $S_{j}$ starting from state $S_{i}$ and if $E_{\text {cell_wlan }}$ is the average number of WLAN entries - from the analytical model this is given by is given by equation 4 .

$\mathrm{E}_{\text {cell_wlan }}=\mathrm{M}_{\mathrm{i}}\left[\mathrm{y}_{\mathrm{j}}^{(\mathrm{k})}\right] \rightarrow\left(\mathrm{z}_{\mathrm{ij}}-\pi_{\mathrm{j}}\right)+\mathrm{k} \pi_{\mathrm{j}}$

Let $\rho$ be equal to the average number of steps taken by a user to reach cell 6 starting from any cell in the 'Location Area'. Then from figure $3 \rho$ is given by

$\rho=\left[4 * \mathrm{M}_{1}\left[\mathrm{y}_{6}{ }^{(\mathrm{k})}\right]+8 * \mathrm{M}_{2}\left[\mathrm{y}_{6}{ }^{(\mathrm{k})}\right]+4 * \mathrm{M}_{3}\left[\mathrm{y}_{6}{ }^{(\mathrm{k})}\right]+4 * \mathrm{M}_{4}\left[\mathrm{y}_{6}{ }^{(\mathrm{k})}\right]+4 * \mathrm{M}_{5}\left[\mathrm{y}_{6}{ }^{(\mathrm{k})}\right]\right] / 24$ (5)

\section{Useful Statistics and Distributions}

For the biased and the unbiased models, the average number of WLAN entries for users starting from different cells in the Location Area of the cellular network and making 20 steps (cell crossings) in a given time (maybe a day) are given in table $1 . \rho$ values provided in the last column were calculated using equation 5 .

In table 2, we have listed the average number of WLAN entries $\rho$ that a user would make if he were to make 5, $10,20,30$ and 40 steps. The readings show a consistent increase in the average number of entries that will be made by the user if he is a highly mobile person. The values of $\rho$ are higher for the biased model as compared to the un-biased model.

$$
\text { TABLE } 1
$$

\begin{tabular}{lllllll}
\multicolumn{7}{c}{ AVERAGE NUMBER OF WLAN ENTRIES } \\
\hline Initial State/cell & 1 & 2 & 3 & 4 & 5 & $\rho(\mathrm{k}=20)$ \\
\hline Unbiased model & 0.349 & 0.421 & 0.485 & 0.622 & 0.870 & 0.528 \\
Biased model & 0.672 & 0.771 & 0.859 & 1.215 & 1.505 & 0.9580 \\
\hline
\end{tabular}

TABLE 2

\begin{tabular}{crrrrr}
\multicolumn{6}{c}{ AVERAGE NUMBER OF WLAN ENTRIES FOR USERS WITH DIFFERENT MOBILITY } \\
\hline K & 5 & 10 & 20 & 30 & 40 \\
\hline (biased model) & 0.27449 & 0.50234 & 0.95803 & 1.4137 & 1.8694 \\
(unbiased model) & 0.17251 & 0.29106 & 0.52816 & 0.76526 & 1.0024
\end{tabular}

Within a LA in a cellular network, the user population would be a mix of users making variable number of steps during their routine daily movement. We have tentatively 'aggregated' the users, as users making 5, 10, 20, 30 and 40 steps. We have then selected a few distributions as a representation of the mixed-user population in a given LA. These are shown in graph 1 . The first distribution 'unil' is a uniform distribution with a high peak; the second uniform distribution 'uni2' is more flat. The next distribution 'hi' reflects predominantly highly mobile users and the last one 'lo' has predominantly low mobile users. A constant distribution 'equal' is also included. Graph 1 gives the distribution plots used to get an estimate on the user mix. Graph 2 is the extra signaling costs (i.e. proportional to the average number of WLAN entries) that will be incurred by the vendor to support handoff for the different user distributions for the unbiased and the biased mobility models.

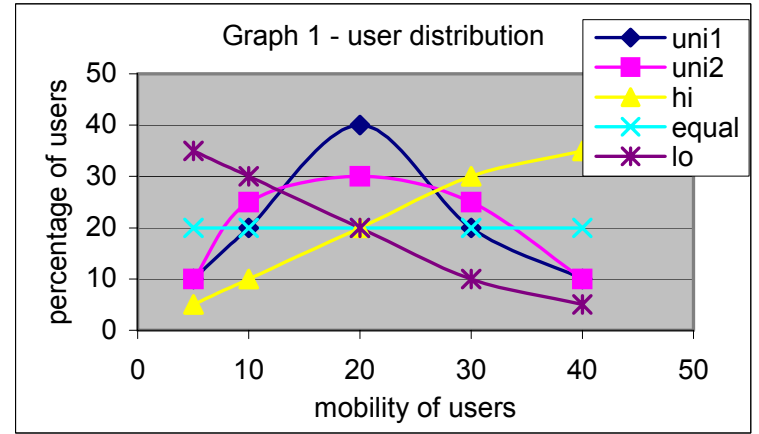

V. Cost Calculations

We introduce the parameters that will be used for cost calculations and in the subsequent sub-section provide the calculations.

\section{A. Parameters for Cost Calculations}

Refer figure 2.

Wireless transmission costs $\quad=c_{1}$

Wired transmission costs for complex messages $\quad=c_{2}$

Wired transmission costs for simple messages $\quad=c_{3}$

Database access/ processing costs at the URAC $\quad=a_{\text {urac }}$

(at points A C)

Database access/ processing costs at the HLR $\quad=a_{\mathrm{hlr}}$

(at point B)

Weight factor for transmission costs $\quad=\alpha$

Weight factor for database processing costs $\quad=\beta$

Average number of entries into the WLAN cell from

anywhere in the LA $=\rho$

Fraction of call time spent in cellular network $\quad=\tau$ units

Call time spent in WLAN $=1-\tau$ units

Cost to the Vendor for call support in cellular network $=\kappa$

Ratio WLAN cost / cellular cost $=\eta$

Cost to the vendor for call support in WLAN $\quad=\kappa^{*} \eta$

Per call cost to the user in cellular network $\quad=\gamma$

Ration of WLAN cost / cellular network cost to the user $=\theta$ 
Call cost to the user in WLAN

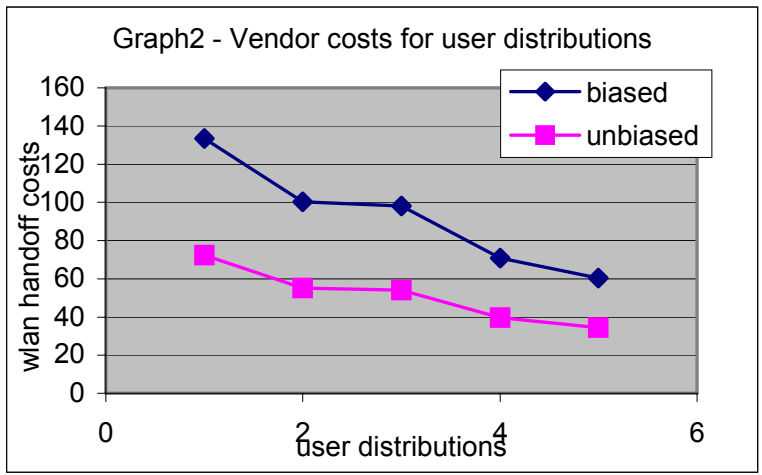

1- hi, 2 - equal, 3- uniform1, 4- uniform2, 5- lo

\section{B. Vendor side Cost calculations}

Infrastructure costs have not been considered. The cost equations provided below are for one user.

Case A: (without the integration)

Call Cost to the vendor (call duration 1 unit time) $=\kappa$

Case B: (with integration)

From figure 2 costs to the vendor for signaling to support a

call across cellular and WLAN is given by $\mathrm{C}_{\text {cell_wlan, where }}$

$\mathrm{C}_{\text {cell_wlan }}=\rho\left[\alpha\left(3 c_{1}+4 c_{2}+9 c_{3}\right)+\left(a_{\text {urac }}+2 a_{\text {hlr }}\right) \beta\right]$

Cost for call support in cellular network $=\tau^{*} \kappa$

Cost for call support in WLAN $=(1-\tau)^{*} \kappa \eta$

From equation 8,9 and 10 , total cost to vendor is given by

Vendor costs $=$ Cost for call support (partly) in cellular + cost for call support (partly) in WLAN + signaling costs

$=\tau^{*} \kappa+(1-\tau)^{*} \kappa \eta+\rho\left[\alpha\left(3 c_{1}+4 c_{2}+9 c_{3}\right)+\left(a_{\text {urac }}+2 a_{\mathrm{hlr}}\right) \beta\right]$

Some tentative values have been assumed for the various parameters just to demonstrate the applicability of the model and to determine when the vendor would cut even if he were to provide call support across cellular and WLAN. The values used are $c_{1}=1.5, c_{2}=0.75, c_{3}=0.5, a_{\text {urac }}=1, a_{h l r}=1.5, \kappa=$ $10, \tau=0.5$ and $\eta=0.05$.

Graph 3 is a plot of the per user cost incurred by the vendor. For comparison, we have provided the cost plot for the "only cellular network" case. The costs to the vendor to support a highly mobile user would be higher, as the probability of his WLAN entry is high. We also notice in the graph that if the message transmissions are weighted more compared to the database costs, then the costs incurred by the vendor is high, because of the number of signaling messages that are exchanged as compared to only three database access. If more time is spent in the cellular network per call i.e. if $\tau$ is higher, then the costs incurred are more - an upward shift in the graph can be noticed. There is a point where the vendor will cut even; this depends on a number of statistics. This cutoff point is of interest to the vendor. The vendor can apply this model and any actual costs to estimate this value.

We have not included the plots for the biased mobility model. Obviously the costs would be higher due to more signaling requirements. Lower values of $\eta$ would make it more attractive for the vendor to support such shared calls.
A more useful statistic would be the costs incurred for the different user population under a given "Location Area". Hence, in graph 4 we plot the costs for the different user populations that we identified in graph 1. For comparison purposes, we have provided the cost plot for the "no WLAN" case. The user distributions are shown in $\mathrm{x}$ axis, the total costs inclusive of call support and signaling costs are given in the $y$ axis. We find that except for the case where $\tau$ is high ( 0.5 and more), and the message transmission costs are heavily weighted, the vendor's total costs will be less with integration than if there had been no integration even if handover signaling costs are included.

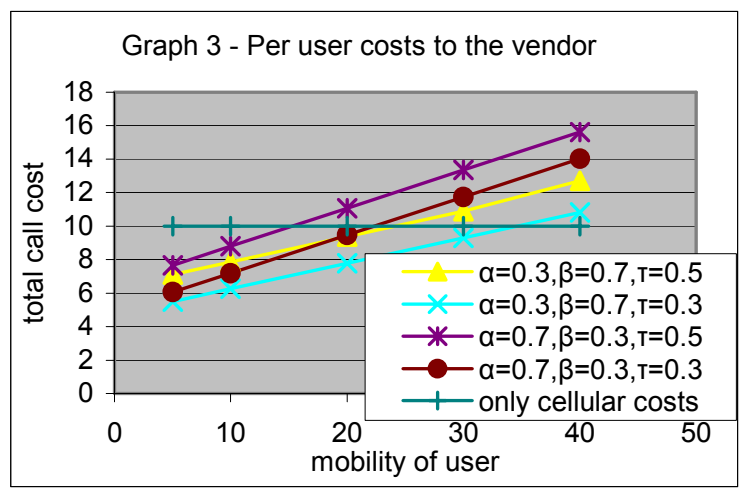

C. User Cost Calculations

We assume a call duration of 1 unit time.

Case A: (without the integration)

Costs - completely supported by cellular networks $=\gamma$

Case B: (with integration)

Call cost in cellular network $=\tau^{*} \gamma$

Call cost in WLAN $=(1-\tau)^{*} \gamma^{*} \theta$

Cost Benefit to the user $=\gamma /\left[\tau^{*} \gamma+(1-\tau)^{*} \gamma^{*} \theta\right]$

Graph 5, gives the cost benefit to a user who has his call supported partly in the cellular network and partly in the WLAN. For the graphs $\gamma$ was maintained at 5 units, $\theta$ was varied from $0.05,0.1$ to 0.3 and $\tau$ was varied from 0.3 to 0.8 in steps of $0.1 . \theta$ was maintained quite high in these calculations to show the extent of benefit to the user even if the cost ratio were not so favorable to the user.

Obviously, the user would benefit even if part of his call were supported in the WLAN. This is clear from the plots in Graph 5, which shows that the more time he spends in the WLAN, the less his total cost. The different plots for various values of $\theta$, show that as $\theta$ increases, i.e. if the WLAN cost is a higher fraction of the cellular cost, the gain to the user is less. Though this scheme benefits the user, in terms of cost, he might face some delays due to the handoff and some voice quality loss due to VoIP.

In the next section, we provide some analysis on the delays that a user can suffer due to the handoffs, especially if there is a heavy traffic at the HLR and the URAC.

\section{Delay Calculations}

Processing time at the databases in milliseconds $\quad=\Gamma$ Average service rate at database in jobs/milliseconds $\quad=\mu$ Average job arrival rate at database in jobs/milliseconds $=\eta$

$\eta_{\text {hlr }}$ - arrival rate of jobs at the HLR, $\eta_{\text {urac }}$ - arrival rate of jobs at the URAC 

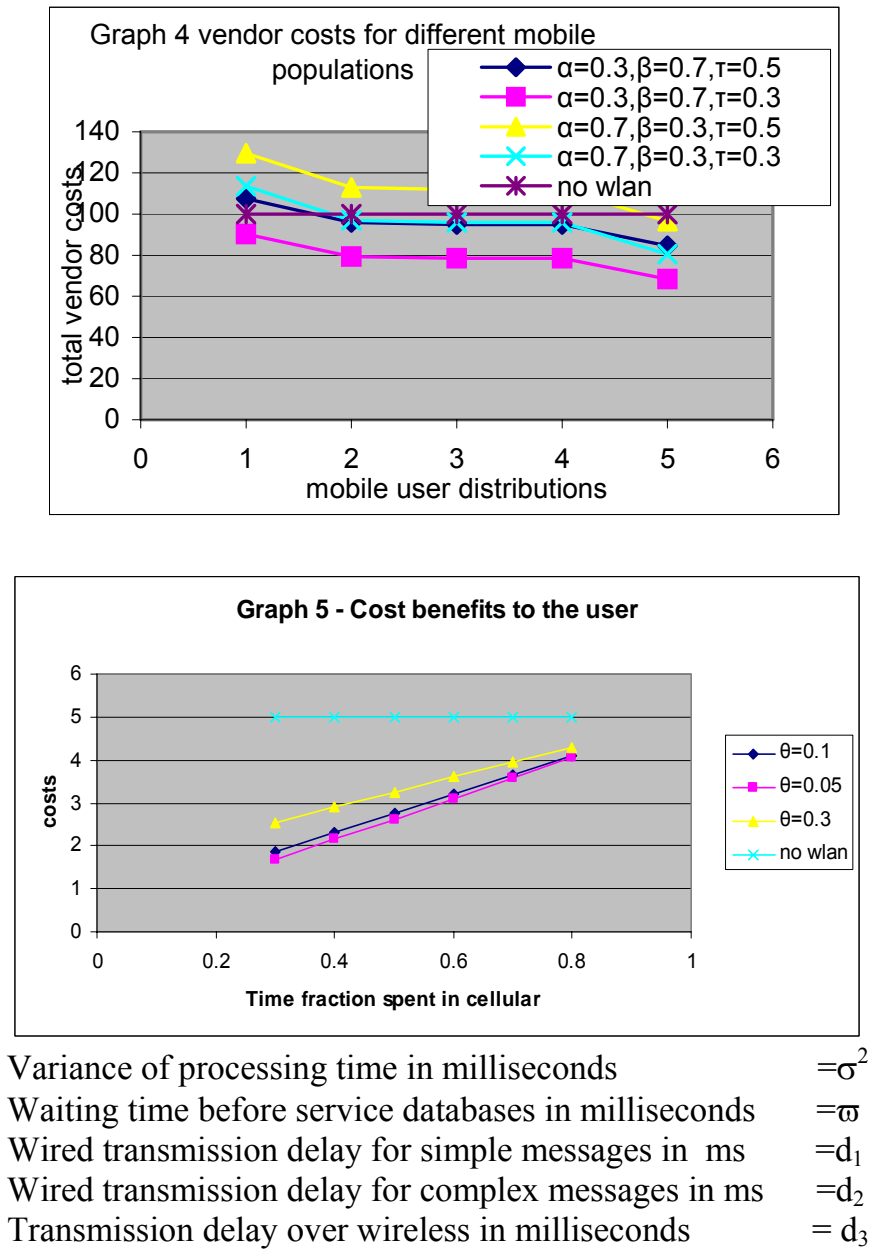

The processing model we have used for all databases is an $\mathrm{M} / \mathrm{G} / 1$ queue [4], where the service time is considered a general distribution and the arrival of jobs Markovian. The total delay for a single handoff process comprises of the system time for all the databases that are involved in the processing plus any transmission delays. The system time in the database in turn comprises the database processing time (or service time) plus the waiting time in the queues. The waiting time in turn depends on the arrival rate of jobs at the database.

For the $\mathrm{M} / \mathrm{G} / 1$ queue system, using the delay parameters mentioned, the system time for a database operation can be obtained using the following equation.

$\Gamma=1 / \mu+\varpi$

where $\varpi$ can be obtained using the Pollaczek-Khinchin [4] equation as given below

$$
\varpi=\frac{\eta \cdot\left(1 / \mu^{2}+\sigma^{2}\right)}{2 .\left(1-\frac{\eta}{\mu}\right)}
$$

From the message exchanges provided in figure 2,

Total Delay $=2 \mathrm{~d}_{3}+10 \mathrm{~d}_{1}+4 \mathrm{~d}_{2}+2 \Gamma_{\text {URAC }}+\Gamma_{\text {HLR }}$

Tentative parameter values used are $-d_{1}=1, d_{2}=1.5, d_{3}=4, \sigma^{2}$ $($ wlan $)=0.04, \sigma^{2}$ (hlr $)=0.4, \mu_{\text {urac }}=1 \mathrm{job} / \mathrm{ms}, \mu_{\mathrm{hlr}}=5 \mathrm{jobs} / \mathrm{ms}$

The 3 plots in graph 6 are for varying values of $\eta_{\mathrm{hlr}}$ for some given values of $\eta_{\text {urac }}$. In the plot $\eta_{-}$hlr $=\eta_{-}$urac, the value of $\eta_{\mathrm{hlr}}=\eta_{\text {urac }}$, because if there is a job arriving at the URAC requesting authentication, then the HLR has to be contacted, assuming that all the requesters are from the same cellular network, which is true in our case. Further, in this plot we have assumed that the only jobs arriving at the HLR are the WLAN crossing and associated authentication requests (in then next graph we have relaxed this assumption). The other two plots assume that there could be more job arrivals at the HLR starting at the minimal value, which is equal to the job arrivals at the URAC. These are the plots where URAC loaded at $50 \%$ and $10 \%$. The delays suffered by the user are plotted for varying loads on the HLR. It is worth noting that the delay effects due to URAC are more profound than the delay effects due to HLR, i.e. because we have provided a higher processing capacity at the HLR, which is normal to assume Graph 7 shows the delays a user may suffer if both the databases are heavily loaded. What we have not included in this study, is the effects of slow links along the signalling path. The delays are not heavy, because "forward" handoff based on the break before make principle was used, which can be accomplished very fast, though at the cost of losing some information. For voice calls such a handoff is ideal, because the QoS requirements for a voice call demands low delays with some acceptable information loss.

\section{CONCLUSIONS AND FUTURE WORK}

We have introduced a cost analysis model to conduct a costing study, which can be used by vendors if they would like to study the benefits of integrating the cellular network and WLAN. Though a number of cases for study can arise under this situation, we have studied the more challenging case of a call continuity support across the two networks. We have restricted the study presented here to the support of voice calls and have made some assumptions, to keep the presentation limited. The model and the equations (with the references provided) however can be used to extend the study to more complex models, hexagonal cells, WLAN occupying more than one cell in the Cellular networks and so on. The study can be used for data connections and the handoff topology modified to accommodate different handoff schemes and their associated signalling.

\section{REFERENCES}

[1] Kuo-Hsing Chiang, Nirmala Shenoy, "A Random Walk Mobility Model for Location Management in Wireless Networks" Proceedings of PIMRC 2001, San Diego.

[2] Kuo-Hsing Chiang, Nirmala Shenoy, "A 2D Random Walk Mobility Model for Location Management in Wireless networks", accepted in IEEE Transactions on Vehicular Technology.

[3] Nirmala Shenoy, "Global Roaming in Next Generation Wireless Networks", Proceedings of CITC3, Rochester NY Sept 19-21, 2002.

[4] Leonard Kleinrock, Queueing Systems, Volume 2, Wiley Interscience

[5] J. G. Kemeny, J. L. Snell, Finite Markov Chains. New York: SpringerVerlag, 1976. 

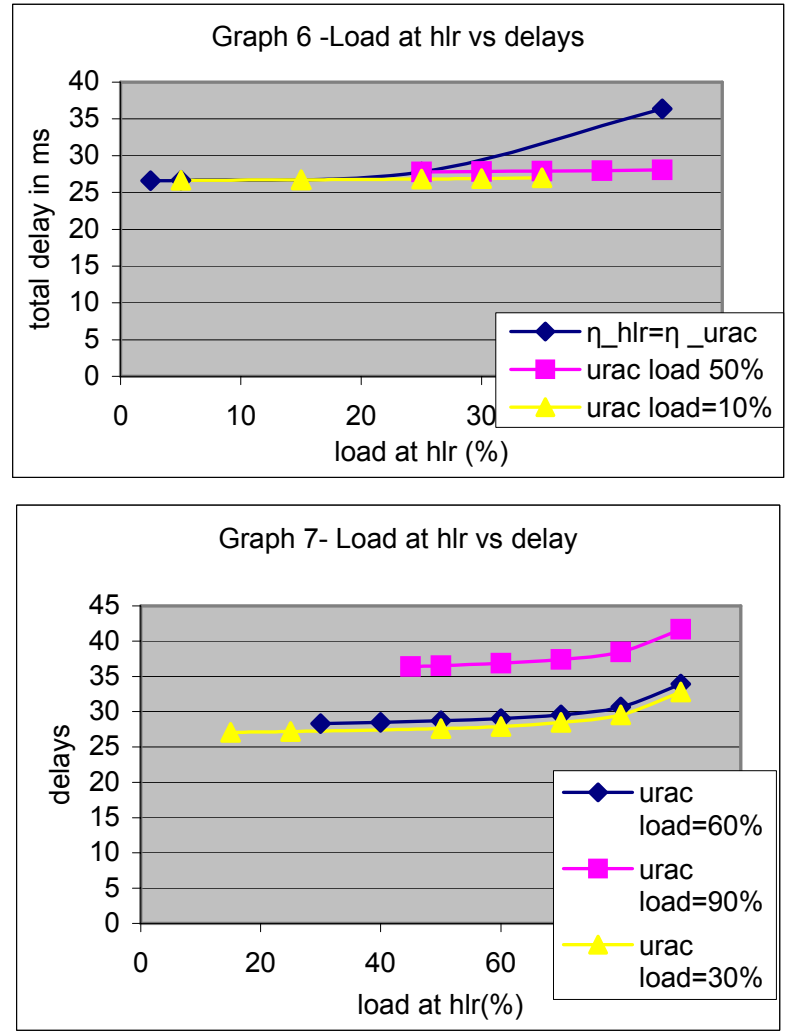